\title{
Introduction: Scientific Discovery and Inference
}

\author{
Emiliano Ippoliti ${ }^{1} \cdot$ Tom Nickles $^{2}$
}

Published online: 3 October 2019

(c) Springer Nature B.V. 2019

Scientific discovery considered broadly as the way new scientific knowledge is generated, or still more broadly as scientific innovation (whether or not a question of true knowledge) has been conceptualized basically in two fashions.

The first one, which we label the non-inferential, goes back at least to the notion of Ancient Greek Muses. The main contention of the non-inferential view is that there is no rational way to account for the way knowledge advances, e.g., by first formulating new hypotheses. A familiar example is Karl Popper, who explicitly states that "there is no such thing as a logical method of having new ideas, or a logical reconstruction of this process" (Popper 1961, p. 32). Many proponents of this view are content to say that discovery is the outcome of 'special processes' that are totally opaque; hence, there is no logical path whatsoever to discovering something new. Notable examples of the non-inferential way are the black box approach (see e.g. Einstein 1958; Popper 1961) and the psychologistic approach (Poincaré 1908). They employ such notions as (Romantic) genius, insight, and intuition to account for discovery. Unfortunately, most of the time, this approach ends up in an obscurum per obscurius, since the concept introduced to explain a seemingly opaque process (discovery, innovation) turns out to be even more opaque.

The second approach, which we label inferential, goes back at least to Plato and Aristotle. It maintains that there is a rational way, or at least a cognitively or rationally intelligible way, to account for the way knowledge advances via new ideas and practices. In its "strong" version, it argues that there is a method (or methods) for discovery, which can be refined, learnt and transmitted. This view can be broken down into at least three approaches: the cognitive approach

Emiliano Ippoliti

emi.ippoliti@gmail.com

Tom Nickles

nickles@unr.edu

Sapienza University of Rome, Rome, Italy

2 University of Nevada, Reno, Reno, USA
(Simon 1977, 1987; Weisberg 2006), the deductive approach (Musgrave 1988, 1989; Zahar 1983), and the heuristic approach (Lakatos 1976; Urbach 1978; Zahar 1983; Nickles 1987, 2006; Cellucci 2013, 2017; Ippoliti 2014, Ippoliti $2017 \mathrm{a}, \mathrm{b})$. It is worth noting here that these approaches do not share the same idea of "inference", or better, they disagree on the kind of inference that can be regarded as a legitimate way of advancing scientific knowledge.

The deductive approach contends that deductive reasoning is the rational way of producing and reconstructing new knowledge. Proponents of other inferential lines reply that this cannot be the case, for the conclusion of a deductive inference, strictu sensu, does not generate new information with respect to its premises. On the other hand, deductive arguments themselves have to be discovered. They do not drop out of the sky for free, else much mathematics (and much other reasoning) would be trivialized. Thus, we have different ways of characterizing the inferential way to discovery.

The historical shift from a non-inferential to an inferential treatment of scientific discovery is discussed by Tom Nickles in his contribution Scientific Discovery as a Topic for Philosophy of Science: Some Personal Reflections, which opens this special issue. In his paper Nickles, one the originators of this shift (see Nickles 1980a, b), provides a retrospective on developments in the treatment of scientific discovery by philosophers, from when this change began in the 1960's and 1970's. This shift, of course, has both theoretical and practical consequences for the way we should reflect upon and $d o$ science. Regarding theoretical consequences, the so-called "friends of discovery" were among the first to call attention to the fact that most scientific practice was omitted by traditional focus on the logic of justification (confirmation theory), including, ironically, concrete justificatory practices. Later, interest in discovery/innovation has been largely absorbed into "the practice turn." Another lesson, both theoretical and practical, is that physics (centrally, mechanics) does not provide the great model for all sciences to emulate-not even many parts of physics itself. There are many different-appropriately different-problem-formulating 
and problem-solving techniques and cultures, not to mention the crucial fact that many innovation-preparing techniques are highly content-specific.

This issue of Topoi explores some inferential paths to scientific discovery and approaches them from both a theoretical and a practical viewpoint. That is, it offers both an analysis of the theories about inferential ways of accounting for scientific discovery and case studies of inferential and heuristic procedures for discovery 'in vivo' in several scientific fields, including mathematics, physics, and engineering.

As concerns the theoretical part, Emiliano Ippoliti, in his paper Scientific Discovery Reloaded, examines how the way scientific discovery has been conceptualized has changed drastically in the last few decades. He shows how its relation to logic, inference, methods, and evolution has been deeply re-structured-'reloaded'. He describes how the logical empiricist and analytical traditions have been challenged by the 'friends of discovery', who opened up not only the way to a rational investigation of discovery but also produced new theories of discovery (like the deductive, cognitive, and evolutionary), as well as new ways of practicing it in cognitively systematic, "rational" ways. Ampliative rules, methods, heuristic procedures, and even logics of discovery have been investigated, extracted, reconstructed and refined. Ippoliti argues that this change triggered a 'scientific discovery revolution': not only a new way of looking at discovery, but also the construction of tools that can guide us to discover something new. He concludes his paper by noting that this is a very important contribution of philosophy of science to science, as it puts the former in a position not only to interpret what scientists do but also to provide and improve tools that they can employ in their activity.

Diana Quarantotto, in her contribution, Aristotle on Science as Problem Solving, offers us a strong argument to look at Aristotle as a founding father of the inferential approach. In more detail, she provides us with an interpretation of Aristotle's view on scientific inquiry as problem solving, that is, as a process that starts from the collected data and moves towards hypotheses, employing several kinds of inferences. To this end, she tackles passages where Aristotle emphasizes the role that the problem-solving activity has in science, and where he describes the history of human problem-solving activity and the historical development of natural science as a problem-solving activity. Then, Quarantotto examines Aristotle's practice of raising, assessing and solving problems as well as the heuristic procedures he employs to move from 'ignorance' to scientific knowledge.

The other great founding father of this approach is Plato, who is the starting point of Carlo Cellucci's paper, Reconnecting Logic with Discovery. Cellucci argues that, according to Plato, the aim of philosophy is to acquire knowledge, and there is a method to do it-a method of discovery. Celluci notes, however, that in the last century this view has been abandoned: the attempt to give a rational account of discovery has been given up, and logic has been disconnected from discovery. Thus, in his paper Cellucci outlines a way of reconnecting logic with discovery and the reasons behind it. He describes the analytic method developed by Plato as the framework for a rational way of accounting for scientific discovery and characterizes ampliative (nondeductive) inference as the necessary addition to it in order to produce plausible hypotheses to solve problems.

Building on Celluci's point about the necessity of ampliative inference, Fabio Sterpetti and Marta Bertolaso observe in their contribution, The Pursuit of Knowledge and the Problem of the Unconceived Alternatives, that when ampliative reasoning is performed, probabilities cannot be assigned objectively. One of the reasons, they note, is that we face the problem of unconceived alternatives: we are unable to explore the space of all possible alternatives to a given hypothesis, because we do not know how this space is shaped. Therefore, if we want to adequately account for the process of knowledge ampliation, we need to develop an account of the process of scientific discovery that is not exclusively based on the probability calculus. Under this light, they maintain that the analytic view of the method of science advocated by Cellucci is interestingly suited to this goal, since it rests on the concept of plausibility. In this perspective, in order to account for how probabilities are in fact assigned in uncertain contexts and how knowledge ampliation is really pursued, they conclude that we have to take into account plausibility-based considerations and look at the outcome of this process, the hypotheses generated to solve a problem, as provisional objects. These hypotheses can very specific and local, as well as general and sometimes a bit metaphysical.

The nature of very general hypotheses about nature is the core of Harold Brown's contribution, that is, Stability and Change in Nature and Science. He focusses on two very general hypotheses about how Nature works and that guide our research, namely stability and change. Brown argues that while change is endemic in nature, scientists nevertheless seek stability amidst change. He observes that, when proposals fail, scientists shift their focus and look for stability elsewhere. After exploring the interaction between stability and change in nature, he concludes that, while the search for stability does meet a human intellectual need, it is nonetheless compatible with our finding what is actually there. For we do not predetermine outcomes of observations and experiments. Bottom line: the belief that there are loci of stability in nature is a working hypothesis; and while it has been fruitful, only future scientific developments can determine if it will continue to hold.

The way we can generate hypotheses to solve a problem is at the center of Tom Nickles's paper, Alien Reasoning: Is a Major Change in Scientific Research Underway? Nickles 
raises a crucial question: are we entering a major new phase of modern science, one in which our standard, human modes of reasoning and understanding, including heuristics, have decreasing value? He notes that, on one hand, the digital revolution (deep connectionist machine learning, big data, cloud computing, simulation, etc.) inspires such claims, but that, on the other hand, they are not new. He notes that the increasing intelligence of machine learning and networking is a deliberately sought, somewhat alien intelligence and, as such, it challenges the traditional, heuristic foresight of expert researchers. Yet he advises that we proceed with caution here, for there is much hype about the new developments in deep learning, and science remains human-centered in important respects.

One way of generating hypotheses to solve a scientific puzzle in an inferential fashion is by employing models, as they provide the basis for shaping the specific content of a chain of inferences whose conclusion is the candidate hypothesis, or a refined working model itself. Thus, it is not surprising that two papers of this issue tackle the role of models in scientific discovery.

In Model-Based Inferences in Modeling of Complex Systems, Miles MacLeod argues that model-based inferences can play a key role in our ability to handle complexity by generating informative, reliable models. He investigates the role of model-based inference in the modern field of computational systems biology and illustrates how these inferences operate, and he analyzes the material and theoretical bases or conditions underlying their effectiveness. So, MacLeod reiterates the significance and centrality of model-based reasoning in day-to-day problem-solving practices, including the role that debugging processes of partial or incomplete models can play in scientific inference and scientific discovery, particularly with respect to complex systems.

In the second paper devoted to models, Scientific Discovery Through Fictionally Modelling Reality, Fiora Salis advances an answer to a long-standing question: how do scientific models 'represent' in a way that enables us to discover new truths about reality and to draw inferences about it? She states that contemporary accounts of scientific discovery answer this question by focusing on the cognitive mechanisms involved in the generation of new ideas and concepts in terms of a special sort of reasoning-or model-based reasoning_involving imagery. In her paper, she advances an alternative answer: she argues for the crucial role of the propositional imagination in the construction and development of models for the purpose of generating hypotheses that are plausible candidates for truth. Specifically, she proposes simple fictionalism as a new account of models conceived as Waltonian games of make-believe. She suggests that models can lead to genuine scientific discovery when they are used as representations that denote real world phenomena and generate two main kinds of theoretical hypotheses, namely, model-world comparisons and direct attributions.

The more theoretical part of this issue of Topoi ends with the paper by Selene Arfini, Tommaso Bertolotti, and Lorenzo Magnani, The Antinomies of Serendipity: How to Cognitively Frame Serendipity for Scientific Discoveries. In this paper, they stress the importance of serendipitous events in scientific frameworks and aim at enriching the philosophical analysis of serendipity by examining some of its most specific features, such as its game-changing effect, the unexpectedness of its occurrence, and its affinity with the concept of "luck". They analyze these characteristics in the light of their cognitive implications in the recognition, performance, and possible stimulation of serendipitous events in relation to scientific discoveries.

The second part of the issue is more about the heuristics approach to the inferential way to discovery, with a focus on innovative research 'in action'. The first paper of this section is provided by Alain Ulazia and Enetz Ezenarro, Helmholtz's Vortex Motion: An Embodied View of Mathematics in the Heuristics of Fluid Mechanics. These authors propose an embodied approach in forming bridges between mathematics and reality, which they develop here by reconstructing the heuristic procedures and inferential work used in fluid mechanics. By means of a historical example on vortex motion by Helmholtz, they show that the intuitive idea of eddy (or vortex) contains cognitive properties of a mental schema, and that it opens the door to many heuristic possibilities (via cooperation with other heuristic tools such as limit thinking, thought experiment, and analogy) for an embodied mathematical explanation of vortex dynamics.

The way a relevant conceptual novelty in mathematics can be produced is the main subject of Emiliano Ippoliti's paper, Manufacturing a Mathematical Group: A Study in Heuristics. Ippoliti examines how the notion of group has been constructed in order to show the kinds of heuristic reasoning that enabled its manufacturing. He analyses salient aspects of the works of Lagrange, Cauchy, Galois and Cayley, in particular the seminal idea resulting from Lagrange's heuristics and how Cauchy, Galois and Cayley developed it. Ippoliti argues that this analysis illuminates how new mathematical entities are generated, and how what counts as a solution to a problem is shaped and changed as research continues. Moreover, he argues that this case study shows us that we have to study what he calls 'inferential microstructures', that is, the ways similarities and regularities are sought, in order to understand how theoretical novelty is constructed and heuristic reasoning is put forward. These micro-structures are the engines for building analogies and other common heuristic devices such as metaphors, similes and simulations, analysis of extreme cases, thought experiments, breaking constraints, and the use of figures, to mention few. 
Analogies, mental simulations, use of figures, and imagery are also the core of John Clement's contribution, Reasoning Patterns in Galileo's Analysis of Machines and in Expert Protocols: Roles for Analogy, Imagery, and Mental Simulation. In this paper, Clement compares reasoning patterns found in Galileo's treatise on machines, On Mechan$i c s$, with patterns identified in case studies of scientifically trained experts thinking aloud, and he identifies many similarities. He shows that, at one level, the primary patterns identified are ordered analogy sequences, with special diagrammatic techniques to support them. At a deeper level, he argues that Galileo develops constructs to describe patterns that can support embodied, imagistic, mental simulations as a central underlying process. Moreover, Clement maintains that a larger, hypothesized pattern of 'progressive imagistic generalization'-Galileo's development of a model or mechanism that becomes more and more general with each machine application while still being imagistically projectable into many machines_-provides a way to think about his progress toward a modern explanatory model of torque. In unpacking Galileo's arguments, Clement offers an appreciation of the Italian's skilful ability to foster imagistic processes underlying scientific thinking.

Moving from one scientific field to another, Mark Thomas Young's paper, Heuristics and Human Judgment: What We Can Learn About Scientific Discovery from the Study of Engineering Design?, examines the use of heuristic reasoning in scientific practice and especially in engineering design. He argues that the successful use of heuristics in engineering design is often considered to depend on a crucial factor that is markedly absent from accounts of the use of heuristics in scientific discovery, namely, experienced judgment. In this paper, he compares attitudes to the role of computer analysis in scientific and engineering practices, with the aim of showing how the limitations of scientific discovery machines reveal the need for including intuition in philosophical accounts of heuristic reasoning in scientific discovery.

The last paper of this special issue, Scientific Discovery and Inference: Between the Lab and Field in Biology, turns to biology for a case study of the inferential approach to discovery. In their paper, Emily Grosholz, Tano Posteraro, and Alex Grigas note that an adequate account of how inferences and discoveries are made in modern biology is a difficult prospect for a philosopher. In effect, biology raises several difficulties: do we really deduce conclusions from Darwin's principles? Once Darwinian biology is integrated with molecular biology, can we deduce the organism from its DNA? What does induction look like in an era where data sets are often too large to be processed by a human being? What is the role of abductive explanatory claims that try to define the biological individual in relation to the microbiome with which it may be associated, or to revise the notion of evolution when the interaction of organism and environment comes to seem much more complex than earlier generations imagined? How should we evaluate "origins of life" experiments conducted in the laboratory, where chemistry shifts to biology and we try to recreate early conditions on earth to which we have no empirical access? How are the carefully controlled conditions in the lab to be brought into productive relationship with the messy, contingent outdoor work of biologists in the field, studying crabs or eelgrass at the marge of the Pacific Ocean, or prairie plants at the edge of a woods on the plains of the Midwest? The authors provide us with complex and interesting answers to these questions, answers that strongly support an inferential approach to scientific discovery.

To conclude, part of the friends of discovery today reject the idea that there is a single 'logic' of discovery or a strictly deductive logic of discovery. Relatedly, a few also reject the idea of "the scientific method", i.e., of a single, general, portable scientific method that governs (or should govern) all of research. Moreover, there remains disagreement about which specific paths to innovation apply to which problem domains. Yet the topic of scientific discovery (and problem solving and innovation more generally), is, by now, securely established as a legitimate subject for philosophical discussion, one on which various approaches to human social and psychological cognition have already cast light.

\section{References}

Cellucci C (2013) Rethinking logic. Springer, Dordrecht

Cellucci C (2017) Rethinking knowledge. Springer, Dordrecht

Einstein A (1958) A testimonial. In: Hadamard J (ed) The psychology of invention in the mathematical field. Dover, Mineola, pp $142-143$

Ippoliti E (2014) Reasoning at the frontier of knowledge. In: Ippoliti E (ed) Heuristic reasoning. Springer, Basel, pp 1-10

Ippoliti E (2017a) Building theories. The heuristic way. In: Dank D, Ippoliti E (eds) Building theories. Springer, Berlin

Ippoliti E (2017b) Heuristic logic. A kernel. In: Danks D, Ippoliti E (eds) Building theories. Springer, Berlin

Lakatos I (1976) Proofs and refutations: the logic of mathematical discovery. Cambridge University Press, Cambridge

Musgrave A (1988) Is there a logic of scientific discovery? LSE Q 2(3):205-227

Musgrave A (1989) Deductive heuristics. In: Gavrovlu K, Goudaroulis Y, Nicolacopoulos P (eds) Imre Lakatos and theories of scientific change. Kluwer, Boston, pp 15-32

Nickles T (ed) (1980a) Scientific discovery: logic and rationality. Springer, Boston

Nickles T (ed) (1980b) Scientific discovery: case studies. Springer, Boston

Nickles T (1987) Lakatosian heuristics and epistemic support. Br J Philos Sci 38:181-205

Nickles T (2006) Heuristic appraisal: context of discovery or justification? In: Schickore J, Steinle F (eds) Revisiting discovery and 
justification: historical and philosophical perspectives on the context distinction. Springer, Dordrecht, pp 159-182

Poincaré H (1908) L'invention mathématique. Enseign Math 10:357-371

Popper K (1961) The logic of scientific discovery. Science Editions, New York

Simon H (1977) Models of discovery. Reidel, Dordrecht

Simon H (1987) Is scientific discovery a topic in the philosophy of science? In: Rescher N (ed) Scientific inquiry in philosophical perspective. University Press of America, Lanham, pp 1-15

Urbach P (1978) The objective promise of a research programme'. In: Radnitzky G, Andersson G (eds) Progress and rationality in science. Reidel, Dordrecht, pp 99-113
Weisberg R (2006) Creativity: understanding innovation in problem solving, science, invention, and the arts. Wiley, Hoboken

Zahar E (1983) Logic of discovery or psychology of invention? Br J Philos Sci 34:243-261

Publisher's Note Springer Nature remains neutral with regard to jurisdictional claims in published maps and institutional affiliations. 